University of Massachusetts Amherst ScholarWorks@UMass Amherst

Masters Theses 1911 - February 2014

1990

\title{
Vasopressin innervation of sexually dimorphic structures of the gerbil forebrain under various hormonal conditions.
}

Bradley J. Crenshaw

University of Massachusetts Amherst

Follow this and additional works at: https://scholarworks.umass.edu/theses

Crenshaw, Bradley J., "Vasopressin innervation of sexually dimorphic structures of the gerbil forebrain under various hormonal conditions." (1990). Masters Theses 1911 - February 2014. 2186.

Retrieved from https://scholarworks.umass.edu/theses/2186

This thesis is brought to you for free and open access by ScholarWorks@UMass Amherst. It has been accepted for inclusion in Masters Theses 1911 -

February 2014 by an authorized administrator of ScholarWorks@UMass Amherst. For more information, please contact

scholarworks@library.umass.edu. 


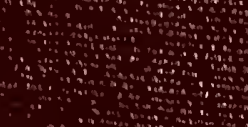




\title{
VASOPRESSIN INNERVATION OF SEXUALLY DIMORPHIC STRUCTURES OF THE GERBIL FOREBRAIN UNDER VARIOUS HORMONAL CONDITIONS.
}

\section{A Thesis Presented}

by

\section{BRADLEY J. CRENSHAW}

\author{
Submitted to the Graduate School of the \\ University of Massachusetts in partial fulfillment \\ of the requirements for the degree of
}

MASTER OF SCIENCE

May 1990

Psychology 
VASOPRESSIN INNERVATION OF SEXUALLY DIMORPHIC STRUCTURES OF THE GERBIL FOREBRAIN UNDER VARIOUS HORMONAL CONDITIONS.

A Master's Thesis Presented by BRADLEY J. CRENSHAW

Approved as to style and content by:

Geest J. De Vries, Chair

$\frac{\text { Qerrale S. Meyer }}{\text { Jetrold S. Meyer, Member }}$

Richard M. Hagen

Richard P. Halgin, Member

Thuenda ie. Torah

Melinda A. Novas, Acting Chairman.

Department of Psychology

ii 


\section{TABLE OF CONTENTS}

\section{LIST OF FIGURES}

Chapter

\section{INTRODUCTION}

Morphological Sex Differences.................................................1

Homology Between Sexually Dimorphic Areas in Rats and Gerbils......................................................

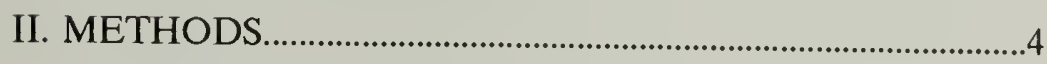

Animals and Treatment..............................................................

Histological Treatment of the Sections....................................4

Quantitative Analysis of the AVP-IR

Fiber Distribution........................................................

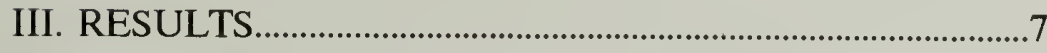

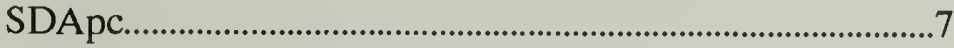

Medial SDA..............................................................................

Lateral SDA..........................................................................

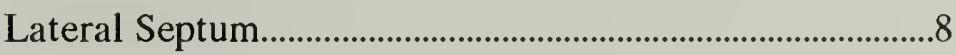

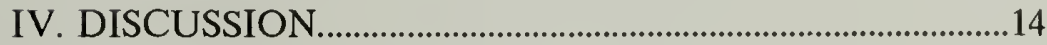

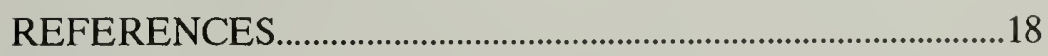




\section{LIST OF FIGURES}

1. Photographs display the sex difference in the innervation of the SDApc in a male (Top) and female (Bottom) gerbil.

2. Graph indicates the sex differences in the size of the clusters of

vasopressin-immunoreactive fibers in the SDApc of males and females.

3. Graph indicates the sex difference in the density of vasopressinimmunoreactive fibers in the medial SDA of males and females

4. Graph indicates differences in the density of vasopressin-immunoreactive fibers in the lateral SDA of males and females $(F 1,25=4.15, P<0.05)$

5. Graph indicates treatment $\left({ }^{* *}\right)$ and sex $\left(^{*}\right)$ differences in the density of vasopressin-immunoreactive fibers in the lateral septum ofmalesandfemales. 


\section{H A P TE R I \\ INTRODUCTION}

\section{Morphological Sex Differences}

The medial preoptic area (MPOA) has been associated with sexually dimorphic behaviors, such as male and female sexual behavior, aggression, and territorial behavior [Ferris, 1986; Yahr, 1987]. Sex differences in the structure of this area have been thought to contribute to the observed functional sex differences. For example, consistent differences have been found in the size of certain subareas in the MPOA, such as the sexually dimorphic nucleus [SDN], which in the rat brain is an area roughly five times larger in males than in females [Gorski, Gordon, Shrine, \& Southam, 1978]. Similar areas have been located in other species, and with a similar sexual dimorphism. The MPOA of adult guinea pigs, ferrets, and humans, for instance, contains a nucleus at roughly the same site as the SDN in rats, that is typically larger in males than in females [Blier, Byrne, \& Siggelkow, 1982; Hines, Davis, Coquelin, Goy, \& Gorski, 1985; Swaab \& Fliers, 1985; Swaab, \& Hofman, 1988; Tobet, Zahniser, \& Baum, 1986]. In gerbils a similar area-the sexually dimorphic area of the MPOA-- in males is distinguished from females by the increased cell density of the area. [Commins \& Yahr, 1984a, Commins \& Yahr, 1984b]. Total volume of the SDA in gerbils is actually similar between the sexes. In the rat, the increased volume of the male appears to be at least partly the function of the increased size of the soma and dendritic extent of the neurons comprising the nucleus, compared with those comprising the female SDN [Hammer \& Jacobson, 1984]. 


\section{Homology Between Sexually Dimorphic Areas in Rats and Gerbils.}

Among the several species in which this area has been studied, the homology between the areas has in most cases not been clearly established. An exception, however, is between rats and gerbils, in which homologies in the sexually dimorphic areas are better understood. In redefining the SDN-POA area on cytoarchitectonic grounds and based on the distribution of serotonin, Simerly and Gorski subdivided the medial preoptic nucleus (MPN) of the rat into medial (MPNm), central (MPNc) and lateral (MPNl) parts [Simerly, Swanson, \& Gorski, 1984]. The MPNc is a particularly cell-dense region that is located within the MPNm, and corresponds to the earlier defined SDN-POA. In gerbils, the male SDA is a hook-shaped structure that also contains an intensely staining cell group, the SDA pars compacta (SDApc). The female SDA appears more ovoid than the male SDA because its medial and lateral components are paler and because the area enclosed by the hook in males has a more distinct ventral border in females. Also, most females lack an SDApc [Commins and Yahr, 1984a]. Acetylcholinesterase staining reveals the same profile of the SDA as Nissl staining. The SDApc, however, lacks acetylcholinesterase activity [Commins and Yahr, 1984b].

Based on structural similarity, position in the brain, and on similar afferent inputs to the area, the SDApc of the gerbil may be homologous to the MPNc of the rat. Both the SDApc in the gerbil and the MPNc in the rat lie roughly midway between the anterior commissure and the optic chiasm [Commins et al, 1984a; Commins et al., 1984b; Gorski et al., 1978]. Both areas also receive significant inputs from the BST [De Vries, Gonzales, \& Yahr, 1988; Simerly \& Swanson, 1986], and both lack acetylcholinesterase activity [Commins \& Yahr, 1984b; Paxinos \& Watson, 1986]. Finally, both the MPNc and 
the SDApc contain a high density of vasopressin-immunoreactive [AVP-IR] fibers [De Vries and Yahr, 1987].

There are differences, however. In particular, the MPNc of rats in adulthood does not appear to be as sensitive to differing levels of sex steroids as is the SDApc of gerbils [Bloch, \& Gorski, 1988]. Further, the distribution of AVP-IR fibers over the SDApc is crisper, and more clearly defined than the distribution observed in the MPNc. In rats, castration does not appear to influence the distribution of AVP-IR fibers in the MPOA. Major effects on AVP-IR fibers are found, however, in other forebrain areas of the rat, where the staining of AVP fibers completely disappears after gonadectomy and reappears after treatment with gonadal steroids [De Vries, Buijs, Van Leeuwen, Caffe, \& Swaab, 1985; De Vries, Duetz, \& Buijs, 1986]. The differences of the SDApc of gerbils and the MPNc of rats--better effects of hormones in adulthood, crisper distribution of AVP-IR fibers over the SDApc in gerbils--suggest that gerbils may be more likely to show hormonal effects on the AVP innervation of this area, which may help to understand better the role of neuropeptides in sexually dimorphic structures that are involved in sexually dimorphic behaviors.

To determine whether the presence of AVP in the gerbil SDA is sexually dimorphic, and whether it is sensitive to levels of gonadal hormones, we compared the density of AVP-IR fibers in adult male and female gerbils. Specifically, we looked at the density of fibers in the medial and lateral SDA and the SDA pars compacta (SDApc). We also measured the density of AVP-IR fibers in the lateral septum--an area whose sex differences are known to be steroid sensitive. 


\section{H A P T E R II \\ METHODS}

\section{Animals and Treatment}

Twenty-four adult gerbils ( 12 males and 12 females) were gonadectomized under chloropent anesthesia, twelve of which (six males and six females) were also implanted with silastic capsules filled with testosterone $(2.5 \mathrm{~cm} ; 1.5 \mathrm{~mm}$ i.d., $2.4 \mathrm{~mm}$ o.d.). An additional twelve animals (six males and six females) received sham gonadectomies and were implanted with empty silastic tubing. Eight weeks after the operations, all animals were deeply anesthetized with chloropent, and perfused through the ascending aorta with $50 \mathrm{ml} 0.9 \%$ saline, followed by $5 \%$ acrolein in $0.1 \mathrm{M}$ phosphate-buffered saline $(\mathrm{pH} 7.2)$ for 5 minutes (about $300 \mathrm{ml}$ per animal). The brains were removed and $50-\mu \mathrm{m}$ thick sections were cut with a vibratome.

\section{Histological Treatment of the Sections.}

Free-floating sections were pre-treated with $1 \%$ sodium borohydride and then incubated with the following solutions:

1). $0.05 \mathrm{M}$ Tris- $\mathrm{HCl}, \mathrm{pH} 7.6$, containing $2 \%$ goat serum, $0.9 \% \mathrm{NaCl}$ and $0.5 \%$ Triton $\mathrm{X}$ 100 (Tris-Triton), 15 minutes rinse;

2). anti-AVP (ICN, Lisle, Il)m 1:2000 in Tris-Triton, overnight at $4^{\circ} \mathrm{C}$;

3). Tris-Triton, 15 minutes rinse;

4). goat-anti-rabbit-IgG (ICN) serum 1:150 in Tris-Triton, 45 minutes;

5). Tris-Triton, 15 minutes rinse;

6). peroxidase-antiperoxidase complex (PAP; ICN) 1:300 in Tris-Triton, 45 minutes;

7). Tris-Triton, 15 minutes rinse. 
After repeating the incubations 4-7, sections were rinsed in $0.5 \mathrm{M}$ Tris- $\mathrm{HCl}, \mathrm{pH} 7.6$, with $0.9 \% \mathrm{NaCl}$ (Tris- $\mathrm{NaCl}$ ), and reacted with $0.05 \%$ 3-3'-diamino-benzidine (DAB) in Tris$\mathrm{NaCl}$ with $0.01 \% \mathrm{H}_{2} \mathrm{O}_{2}$, for 20 minutes. After a rinse in Tris- $\mathrm{NaCl}$, sections were mounted on slides, air dried, and cover-slipped. Every fourth brain section of each animal was thionin stained. These sections were used to identify the site of the SDApc.

\section{Quantitative Analysis of the AVP-IR Fiber Distribution}

Sex differences and the effects of hormonal manipulations on the density of AVPIR fibers were studied in 1). the medial SDN; 2). the lateral SDN; 3) the SDApc and 4).the lateral septum by computerized image analysis using a Microcomputer Imaging Device (MCID) of Imaging Research Inc. (St. Catherines, Ontario). For this, the same coded sections as those used in the independent rating were analyzed. Fiber density was expressed as the proportion of a sampling area that was covered by AVP-IR fibers in the $50 \mu \mathrm{m}$-thick sections. This proportional area was determined by digitizing the image of a section into an array of pixels with gray values ranging from 0 to 255 . A binary picture of that image was then obtained by displaying the figure into two colors, one corresponding to pixels with gray values below a variable set point, and the other corresponding to pixels with gray values equal to or above that set point. This set point was established at the level at which fibers appeared in one color, and background in another (gray-level thresholding; Shipley, Luna, and McLean, 1989]. The ratio of the number of pixels displaying the fibers to the number of all pixels in the sampling area was taken as the proportional area covered by the AVP-IR fibers in the particular brain section being analyzed.

In the SDApc, the sample area consisted of the cluster of AVP-IR fibers that covered the pars compacta. The computer was used to draw a perimeter around the area 
that contained the heaviest innervation of AVP-IR fibers in the SDApc, after which fiber density within that area was established as above. We took the area within the perimeter as the size of the cluster of AVP-IR fibers.

Because there are no major sex differences or hormonal effects on the size of the the medial and lateral SDA and the lateral septum, our sample areas of these structures were defined differently. For both the medial and the lateral SDA, two alternate sections at the center of each structure was chosen for every animal. A square of $80 \times 80 \mu \mathrm{m}$ situated in the center of these structures was analyzed bilaterally. For the lateral septum, which contains a more extended area of AVP innervation, the same was done in four connected and vertically arranged squares in two alternate sections at the level where the anterior commissure crosses the midline. For every animal, the means of all measures sampled from the medial and lateral SDA, and the lateral septum were calculated and used as the measures of the AVP-IR fiber density in each respective brain area. Sex differences in and hormonal effects on fiber density were tested with ANOVA. If differences were found, individual differences between different treatment groups were tested with the two-tailed $\mathrm{T}$ test. 


\section{H A P T E R III}

\section{RESULTS}

Although the gerbils survived the surgery, in the course of eight weeks between gonadectomy and sacrifice three animals died. The distribution in the gerbil of AVP innervation in the medial preoptic area and in the lateral septum resembles the distribution seen in rats over the same brain areas, except that in gerbils there is a crisper distribution of AVP-IR fibers in the SDApc than is observed in rats.

\section{$\underline{\text { SDApc. }}$}

Clustering of AVP-IR fibers was found in both sexes in the area that in males houses the SDApc. An ANOVA demonstrated that there are sex differences in the size of these clusters $(\mathrm{F} 1,25=43.73, \mathrm{P}<0.0001]$. In every treatment group, males had larger clusters than did females (Figure 1). There were no significant sex differences in density of AVP-IR fibers within these clusters (Figure 2). There was, however, a significant interaction of sex and treatment $(F 2,25=4.44, P<0.0223)$.

\section{Medial SDA.}

Sex differences were also found in the medial SDA, in which males in every treatment group had significantly greater density of AVP-IR fibers than females $(F 1,25=11.04, P$ $<0.0027)$. There were no statistically significant treatment effects. However, a trend was observed in both males and females, in which fiber density increased after gonadectomy. Treatment with testosterone did not reduce fiber densities to that of controls (Figure 3).

\section{Lateral SDA.}

The presence of AVP-IR fibers in the lateral SDA was scant in both males and females in all treatment groups. However, analysis did indicate treatment differences $(\mathrm{F} 1,25=$ $4.15, \mathrm{P}<0.0278)$. Gonadectomy decreased the amount of AVP-IR fiber staining in both 
males and females when compared to sham-operated animals, and testosterone treatments returned staining densities to that of controls (Figure 4).

\section{Lateral Septum.}

The staining patterns observed in the lateral septum resemble those seen in rats. Different hormonal treatments caused significant differences in staining density of AVP. IR fibers in the lateral septum of both males and females $(F 2,24=59.63, \mathrm{P}<0.0001)$. Gonadectomy significantly decreased AVP fiber staining in both males and females, and testosterone treatments restored fiber density to control levels (Figure 5). There was also a significant difference in the fiber density between males and females $(F 2,24=$ $19.50, \mathrm{P}<0.0002)$, males having more than twice as dense a fiber innervation in the sham-operated and testosterone-treated group (See Figure 4). 

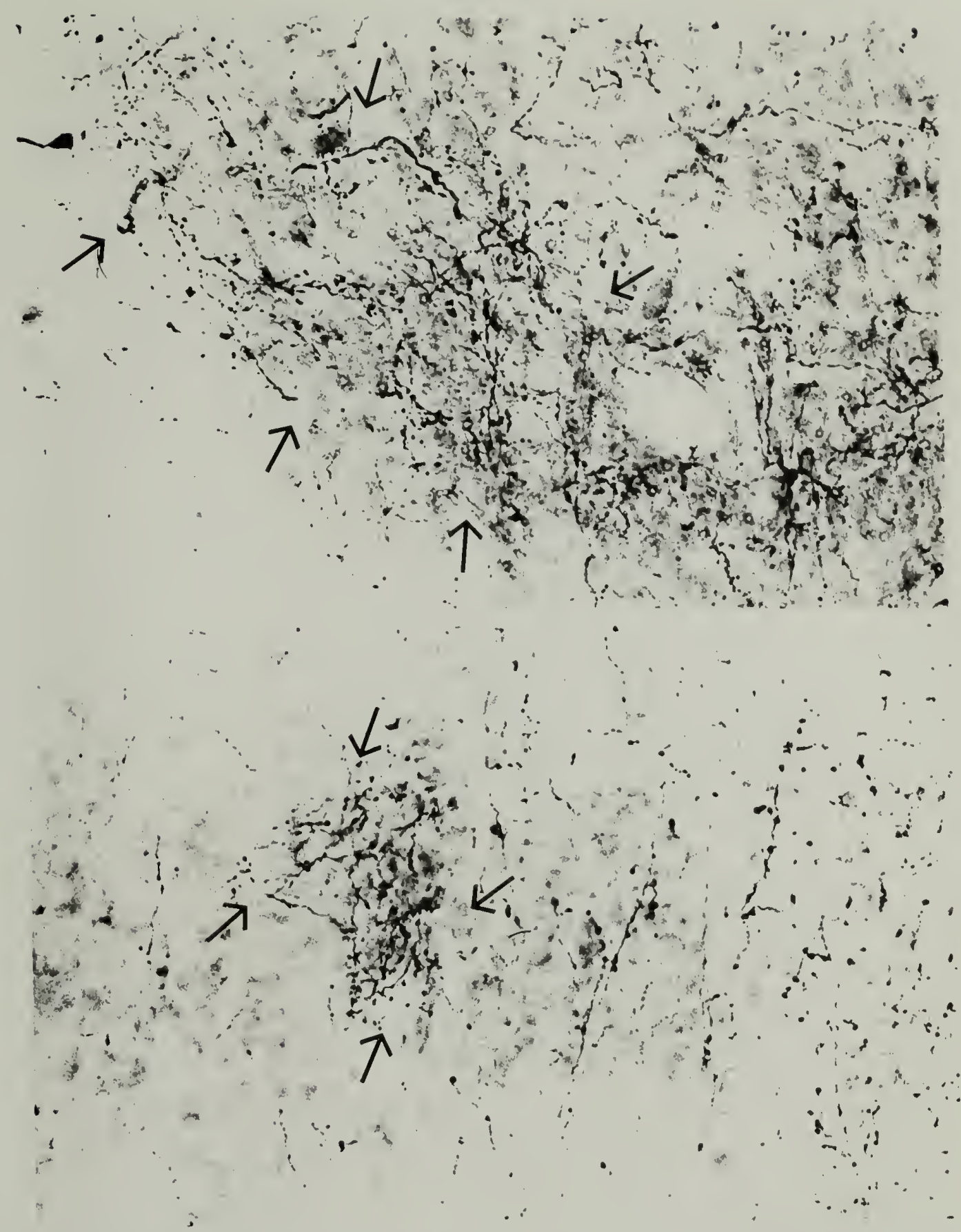

Figure 1: Photographs display the sex difference in the innervation of the SDApc in a male (Top) and female (Bottom) gerbil. Note that the cluster of vasopressinimmunoreactive fibers in the SDApc of the male is larger (Arrows) than the cluster of AVP-IR fibers in the female (Arrows). 


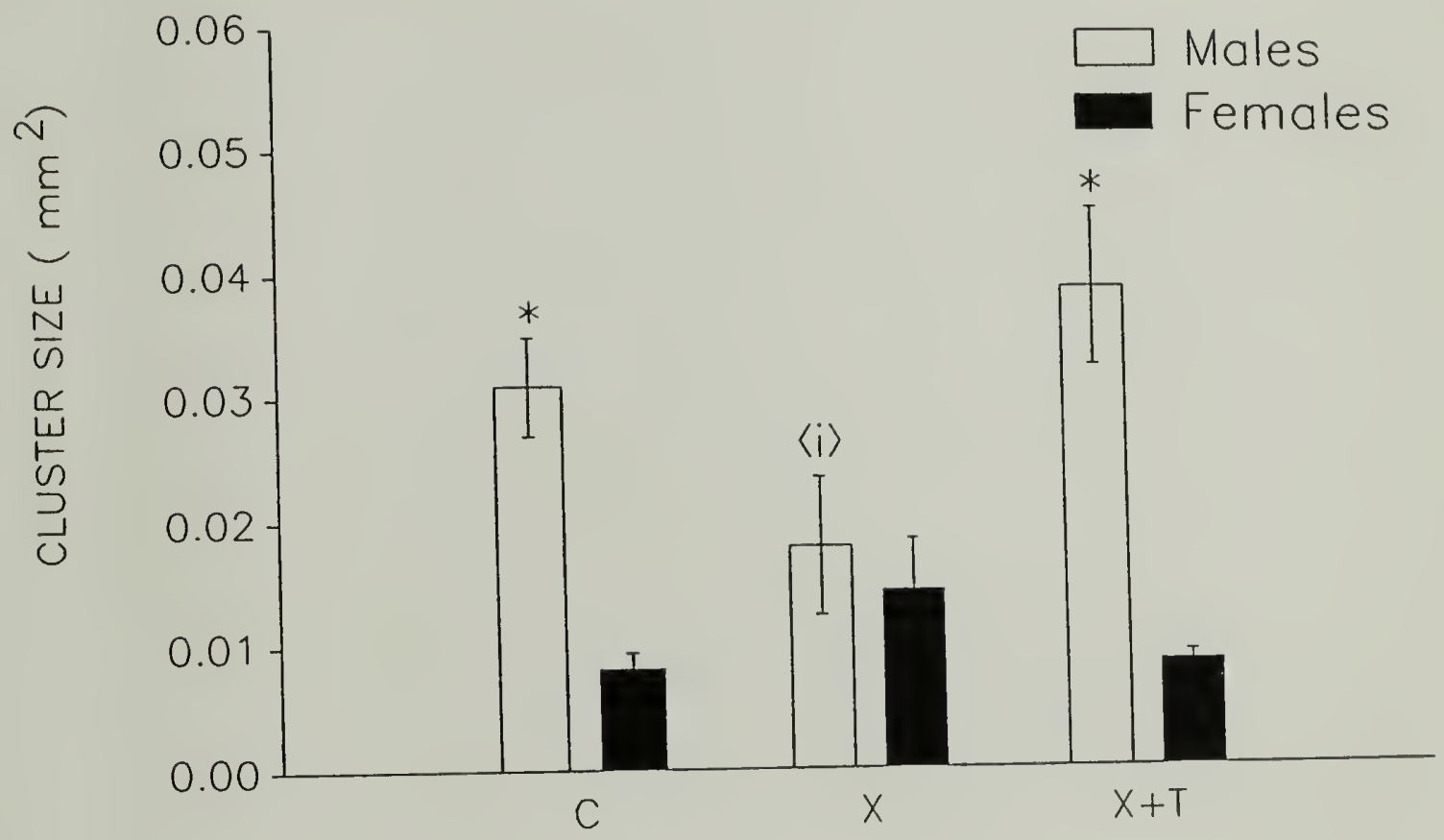

Figure 2: Graph indicates the sex differences in the size of the clusters of vasopressinimmunoreactive fibers in the SDApc of males and females. Asterisk indicates statistically significant differences between males and females in the sham-operated group $(\mathrm{P}<0.0005)$ and in the group of gonadectomized animals receiving testosterone $(\mathrm{P}<0.001) ;<\mathrm{i}>$ indicates an significant interaction of sex and treatment $(\mathrm{P}<0.05)$. 


\section{medial SDA}

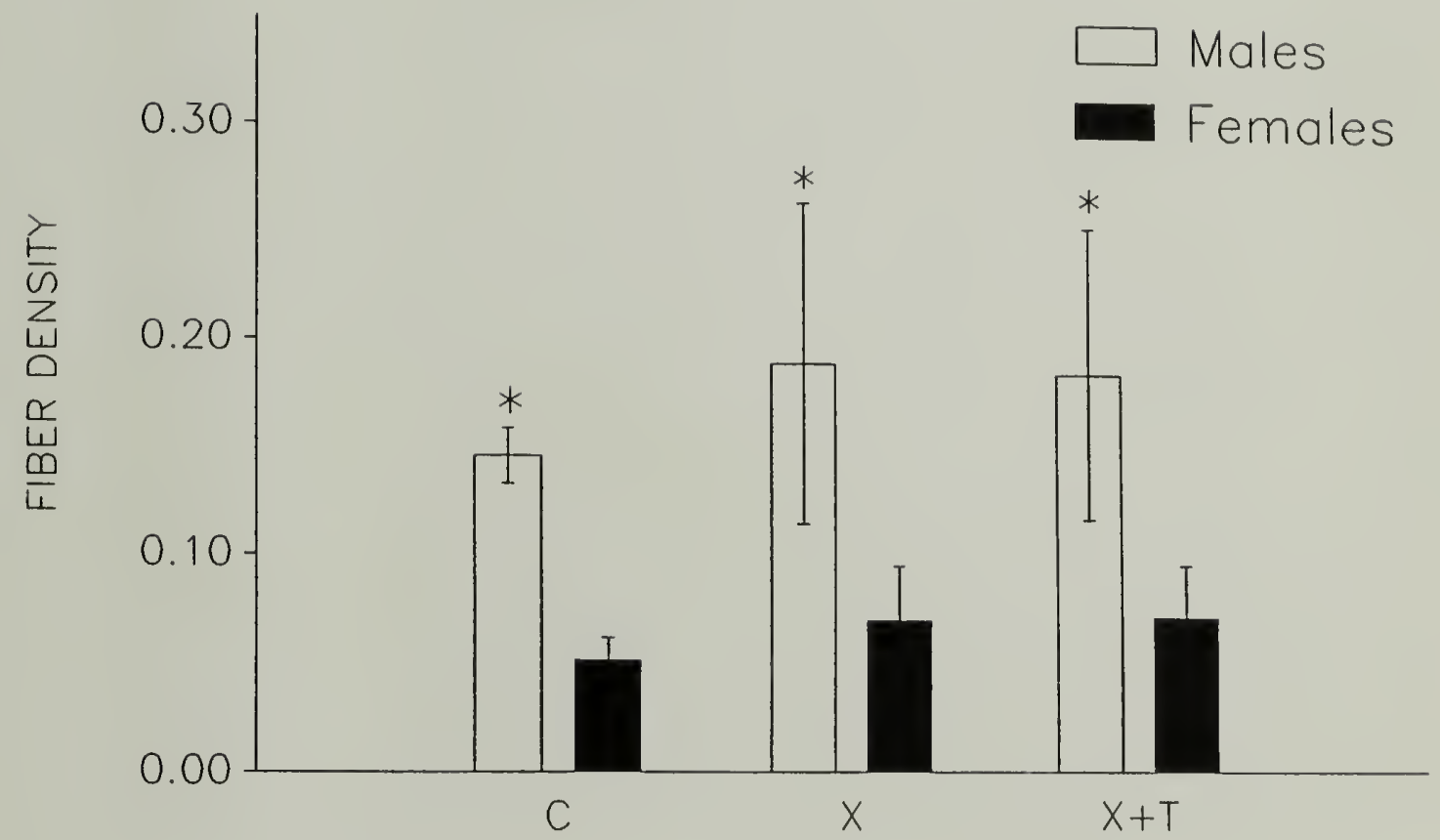

Figure 3: Graph indicates the sex difference in the density of vasopressin-immunoreactive fibers in the medial SDA of males and females. Asterisks indicate statistically significant differences between males and females in every treatment group $(\mathrm{P}<0.005)$. 


\section{lateral SDA}

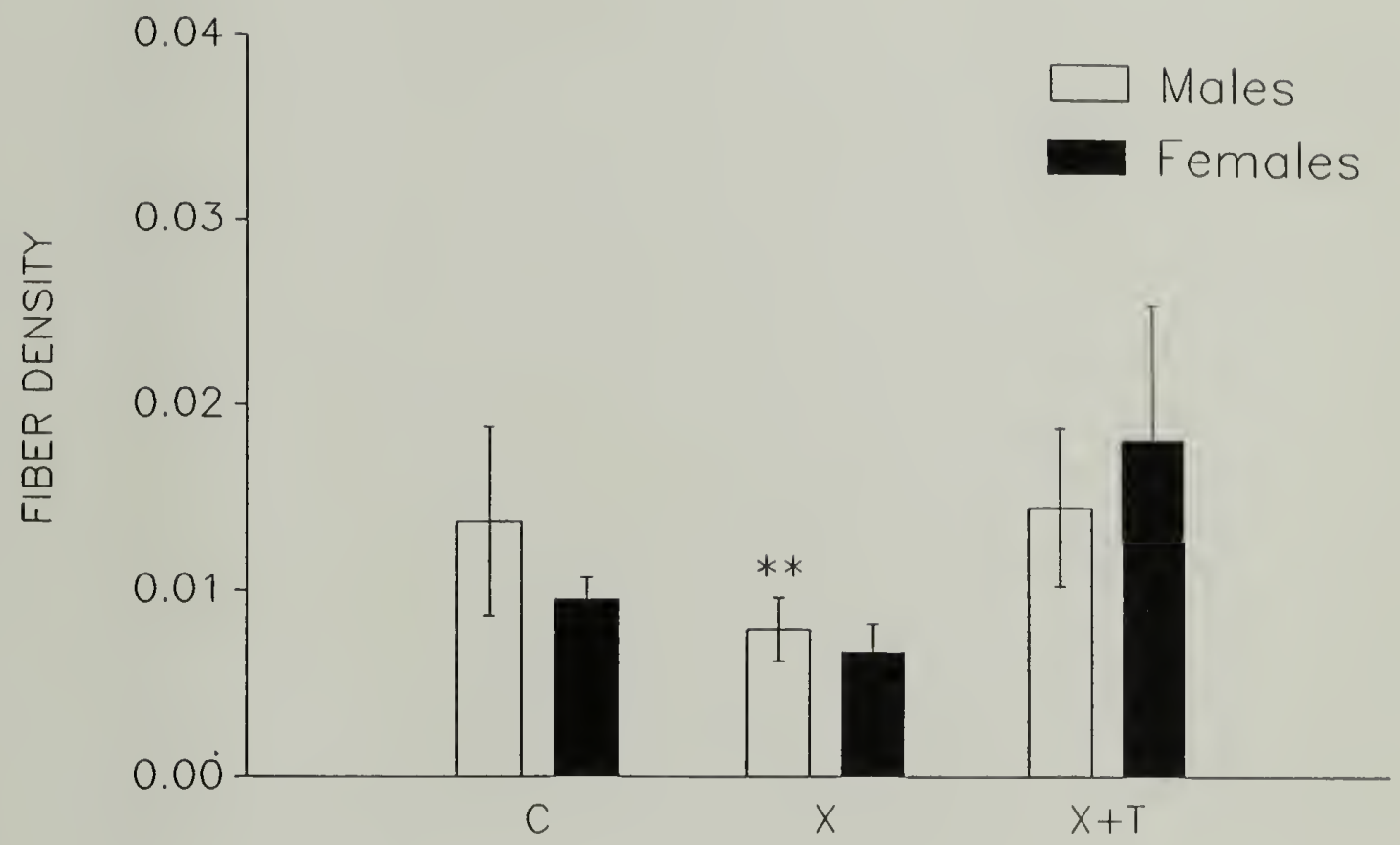

Figure 4: Graph indicates differences in the density of vasopressin-immunoreactive fibers in the lateral SDA of males and females $(\mathrm{F} 1,25=4.15, \mathrm{P}<0.05)$. Double asterisks indicates statistically significant treatment differences between gonadectomized animals and the sham-operated animals. Testosterone restores fiber densities. Note there are no sex differences. 


\section{lateral septum}

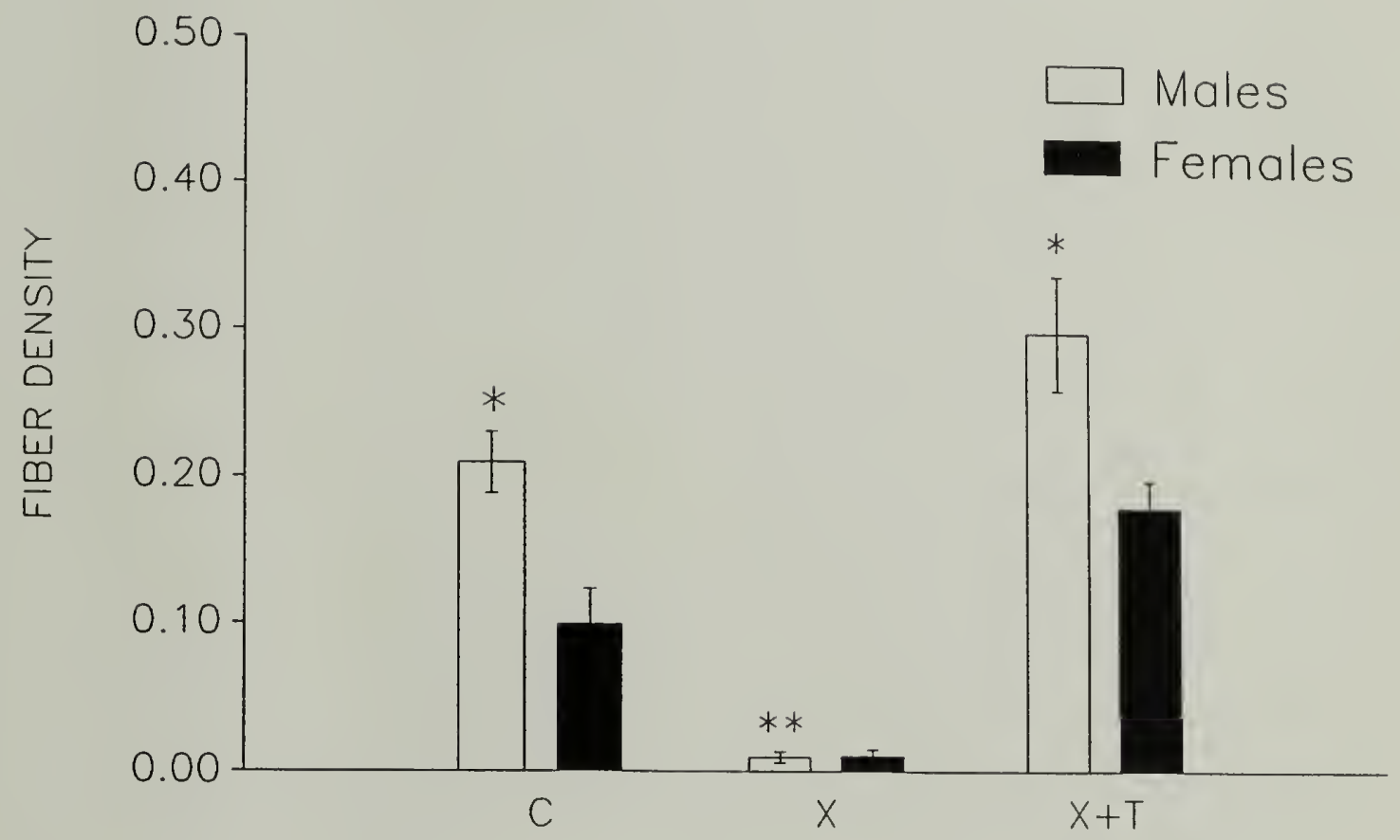

Figure 5: Graph indicates treatment $\left({ }^{*}\right)$ and sex $\left({ }^{*}\right)$ differences in the density of vasopressin-immunoreactive fibers in the lateral septum of males and females. In the sham-operated and testosterone-treated groups, males had a denser innervation than had females $(\mathrm{P}<0.01$ and $\mathrm{P}<0.05$, respectively). Castration reduced the fiber density in both males and females $(\mathrm{F} 1,24=59.63, \mathrm{P}<0.0001)$. 


\section{CHA P TE R IV \\ DISCUSSION}

The major finding of this study is that the AVP innervation of both the SDApc and the medial SDA of gerbils is sexually dimorphic. In males the area occupied by the cluster of AVP-IR fibers covering the SDApc is larger than in females. Similarly, male brains show a higher fiber density in the medial SDA than is found in female brains. Interestingly, unlike the AVP innervation of the lateral septum, where AVP staining disappeared after gonadectomy, the AVP innervation of neither of these two structures appear to be strongly influenced by gonadal hormones in adulthood.

Further, there were two interesting discrepancies between the structures visible in the Nissl staining, and those apparent in the AVP staining. First, when using a Nissl stain, there is a significant sexual dimorphism in the SDApc: thionin staining reveals the presence of the structure in males, and the absence of the structure in females [Commins and Yahr, 1984]. However, when stained for AVP, a concentration of AVP-IR fibers in the SDApc is disclosed in females as well as males, though there remains a sexual dimorphism in both the size and fiber density of the structure.

Second, the size and density of the SDApc and the medial SDA in male gerbils, when stained with thionin, are influenced by gonadal hormones: the intensity of thionin staining in the male SDApc and the medial SDA decreases in castrated animals. The concentrations of AVP-IR fibers in these structures, however, were not influenced by gonadectomy.

The different responses to hormonal conditions among the several brain areas included in this study suggest that the origin of the AVP-IR fibers in the medial and 
lateral SDA, and the SDApc may differ from the origin of the fibers in the lateral septum. At least one retrograde study has demonstrated that the medial SDA receives its primary inputs from the medial part of the BST, the ventral part of the lateral septum, and the medial amygdala, which in rats form the source of the AVP-IR fibers in the lateral septum [De Vries and Buijs, 1983; De Vries et al., 1988]. However, if the medial SDA and the SDApc were solely innervated by steroid-sensitive projections from the BST, the MA and the lateral septum, then decreases in fiber density and area size in the medial SDA and SDApc might be expected. These decreases were not found. Indeed, a trend was observed in the medial SDA in which fiber density increased in gonadectomized males and females, which is the opposite effect observed in the lateral septum and the lateral SDA. Further, among females, the size of the cluster of AVP-IR fibers in the SDApc increased after gonadectomy; testosterone treatment appears to reduce the size of the structure to that of controls. It would be interesting to study fluctuations of AVP fiber staining over the estrous cycle.

It may be that the medial SDA and SDApc receive their AVP-IR fibers from AVP cells in the BST and medial amygdala that respond differently to gonadal hormones than those that project to the lateral septum. On the other hand the innervation of these areas might come from an area at the dorsal border from the SCN where many cells could be labeled retrogradely from the medial SDA [De Vries et al., 1988] and where we found many AVP-IR cells (Unpublished results obtained during this study]. A third possibility is that the medial SDA and SDApc receive a combined input from the BST and the SCN. If this is true and cells in these two areas would respond differently to hormonal manipulations the changes seen in the medial SDA and SDApc might be a combination of the effects on both systems. 
These findings may contribute to a better understanding of the physiological role AVP plays in sexual behavior. The sensitivity to steroids of AVP innervation of the lateral septum, the lateral SDA, and perhaps the medial SDA suggests that AVP in these areas is involved in behaviors that are influenced by gonadal hormones, for example territorial behavior. Lesion studies have shown that scent marking behavior in male gerbils are disrupted when the medial SDA is ablated [Commins \& Yahr, 1984c]. Comparative data support an involvement of AVP in marking: the flank marking behavior of hamsters can be elicited by injecting AVP in the preoptic area, and levels of AVP in the medial preoptic area (MPOA) and the anterior hypothalamus (AH), which are in turn influenced by the presence or absence of testosterone, correlate with levels of mating behavior [Albers, Liou, \& Ferris, 1988]. Behavioral manipulations can similarly influence levels of AVP in the MPOA and the AH. In subordinate male hamsters, AVP immunoreactivity was shown to decrease in subordinate male hamsters when they were exposed repeatedly to dominant aggressive males [Ferris, Axelson, Martin, \& Roberge, 1989]. It is unclear, however, whether there are differences in AVP levels in the SDA of gerbils that mark more or less.

The lateral SDA receives its inputs chiefly from the rostrodorsal part of the lateral septum, the lateral part of the BST, and the anterior and posterior hypothalamic areas [DeVries, et.al., 1988]. As the innervation in this area shows a decrease after gonadectomy and a reappearance after testosterone treatment of AVP staining, these fibers might be derived from the same source as the fibers in the lateral septum. In addition to differences in the effects of lesions of the medial and lateral SDA, and in the afferents of these areas [Commins and Yahr, 1984; De Vries et al., 1988], this is yet another indication that the lateral and medial SDA are functionally different structures. 
Finally, in addition to influencing steroid-dependent functions in adulthood, AVP has been implicated in influencing development. Brattleboro rats, for example, show a stunted brain development [Boer, Swaab, Uylings, Boer, Buijs, \& Velis, 1980; Williams, Carter, \& Lightman, 1985], and recently Bernroider (1988) has demonstrated that there is no significant sex difference in the size of the SDN in rats. The abundance of AVP fibers in the most extremely sexually dimorphic areas of the hypothalamus may be a testament to a role for AVP in the sexual differentiation of this area. 


\section{REFERENCES}

Albers, H.E., Liou, S.Y., \& Ferris, C.F. (1988). Testosterone alters the behavioral response of the medial preoptic-anterior hypothalamus to microinjection of arginine vasopressin in the hamster. Brain Res. 456: 382-386.

Bernroider, G. (1988). Cell-patterning during sexual neurogenesis. Prog. in Neurobio. 31: 477-506.

Blier, R., Byrne, W., \& Siggelkow, I. (1982). Cytoarchitectonic sexual dimorphisms of the medial preoptic and anterior hypothalamic areas in guinea pig, rat, hamster, and mouse. J.Comp. Neurol. 212:118-130.

Bloch, G.J., \& Gorski, R.A. (1988). Cytoarchitectonic analysis of the SDN-POA of the intact and gonadectomized rat. J. Comp. Neurol. 275:604-612.

Boer, G.J., Swaab, D.F., Uylings, H.B.M., Boer, K., Buijs, R.M., and Velis, D.N. Neuropeptides in brain development. In P. McConnell, G.J. Boer, N.E. Van de Poll, H.R. Romijn and M.A. Corner (Eds), Adaptive Capabilities of the Nervous System, Progress in Brain Research, Vol 54, Elsevier/North-Holland, Amsterdam, 1980.

Commins, D. \& Yahr, P. (1984a). Lesions of the sexually dimorphic area disrupt mating and marking in male gerbils. Brain. Res. Bull. 13: 185-193.

Commins, D. \& Yahr, P. (1984b). Acetylcholinesterase activity in the sexually dimorphic area of the gerbil brain: Sex differences and influences of adult gonadal steroids. J. Comp. Neurol. 224: 123-131.

Commins, D. \& Yahr, P. (1984c). Adult testosterone levels influence the morphology of a sexually dimorphic area in the mongolian gerbil brain. J. Comp. Neurol. 224:132-140.

De Vries, G.J., \& Al-Shamma, H.A. (1990). Sex differences in hormonal responses of vasopressin pathways in the rat brain. J. Neurobiol. In press.

De Vries, G.J., \& Buijs, R.M. (1983). The origin of the vasopressinergic and oxytocinergic innervation of the rat brain with special reference to the lateral septum. Brain Res. 273:307-317.

De Vries, G.J., Buijs, R.M., Van Leeuwen, F.W., Caffe, A.R., \& Swaab, D.F. (1985). The vasopressinergic innervation of the brain in normal and castrated rats. J. Comp. Neurol. 233:236-254.

De Vries, G.J., Duetz, W., Buijs, R.M., Van Heerikhuise, J., \& Vreeburg, J.T.M. (1986). Effects of androgens and estrogens on the vasopressin and oxytocin innervation of the adult rat brain. Brain Res. 399:296-302 
De Vries, G.J., Gonzales, C.L., \& Yahr, P. (1988). Afferent connections of the sexually dimorphic area of the hypothalamus of male and female gerbils. J. Comp. Neurol. 271:91-105

De Vries, G.J. \& Yahr, P. (1987). Vasopressin innervation of sexually dimorphic structures in rat, gerbil and human brains. Soc. Neurosci. Abs. 13: 1164.

Ferris, C.F., Axelson, J.F., Martin, A.M., \& Roberge, L.F. (1989). Vasopressin immunoreactivity in the anterior hypothalamus is altered during the establishment of dominant/subordinate relationships between hamsters. Neurosci $\underline{29}(3)$ : 675683.

Gorski, R.A., Gordon, J.H., Shryne, J.E., \& Southam, A.M. (1978). Evidence for a morphological sex difference within the medial preoptic area of the rat brain. Brain Res. 148:333-346.

Hammer, R.P., \& Jacobson, C.D. (1984). Sex difference in dendritic development of the sexually dimorphic nucleus of the preoptic area in the rat. Int. J. Devl. Neuroscience 2(1):77-85.

Hines, M. Davis, F.C., Coquelin, A., Goy, R.W., \& Gorski, R.A. (1984). Sexually dimorphic regions in the medial preoptic area and the bed nucleus of the stria terminalis of the guinea pig brain: A description and an investigation of their relationship to gonadal steroids in adulthood. J. Neurosci. 5(1): 40-47.

Paxinos, G. \& Watson, C. (1986). The rat brain in stereotaxic coordinates. North Ryde, Aust.: Academic Press.

Shipley, M.T., Luna, J., and McLean, J.H. (1989) Processing and analysis of neuroanatomical images. In: Neuroanatomical Tract Tracing Methods 2. Heimer, L., Záborsky, L., (eds.) pp. 331-390.

Simerly, R.B. \& Swanson, L.W. (1986). The organization of neural inputs to the medial preoptic nucleus of the rat. J. Comp. Neurol. 225: 312-342.

Simerly, R.B., Swanson, L.W., \& Gorski, R.A. (1984). Demonstration of a sexual dimorphism in the distribution of serotonin-immunoreactive fibers in the medial preoptic nucleus of the rat. J. Comp. Neurol. 225:151-166.

Swaab, D.F., \& Fliers, E. (1985). A sexually dimorphic nucleus in the human brain. Science 228:1112-1115.

Swaab, D.F., \& Hofman, M.A. (1988). Sexual differentiation of the human hypothalamus: Ontogeny of the sexually dimorphic nucleus of the preoptic area. Dev. Brain Res. 44:314-318. 
Tobet, S.A., Zahniser, D.J., \& Baum, M.J. (1986) Sexual dimorphism in the preoptic/anterior hypothalamic area of ferrets: Effects of adult exposure to sex steroids. Brain Res.364:249-257.

Williams, T.D.M., Carter, D.A., \& Lightman, S.L. (1985). Sexual dimorphism in the posterior pituitary response to stress in the rat. Endocrin. 116: 738-740. 


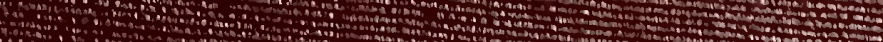

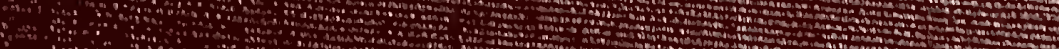

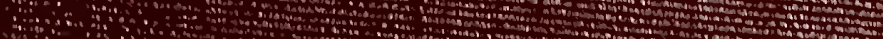

4. 4 a $\therefore$. A 30 -

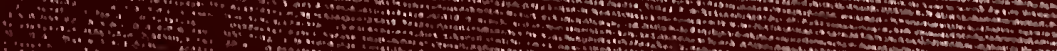

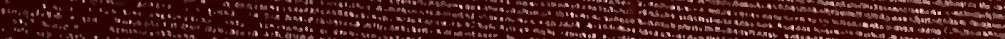

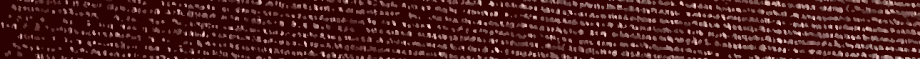

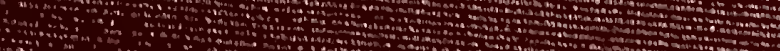

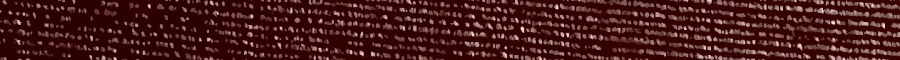

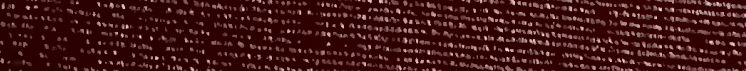
and

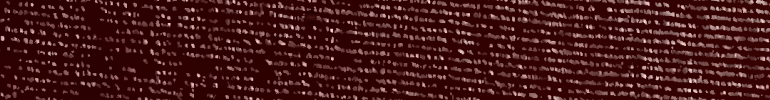

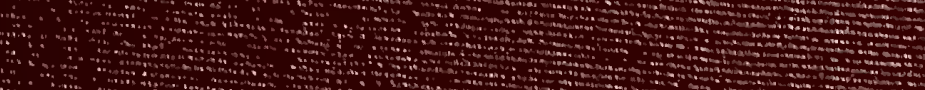
a d

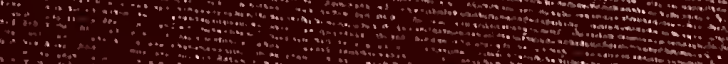

(1) a d a d (1) 40

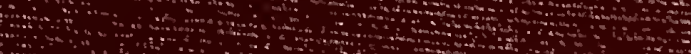

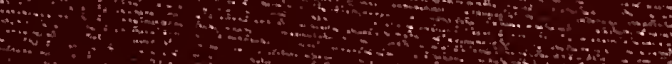

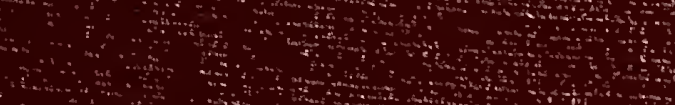
: $:$ $\therefore \therefore$

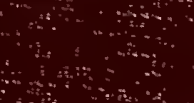

(1)

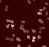

a A 0

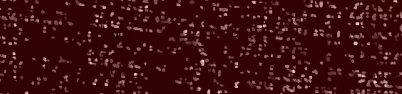
40 H. 40 4

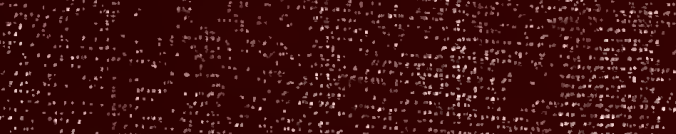
a d a d t

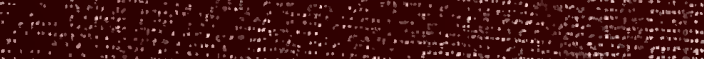

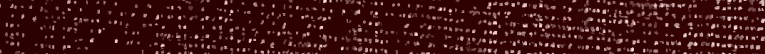
(1) 4 a d a d .

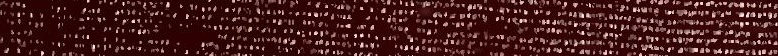
10

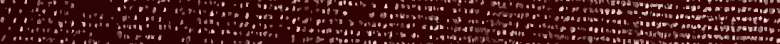
…

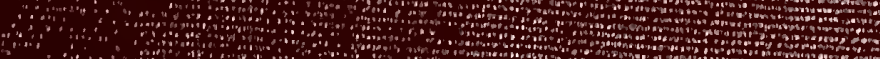
$A$ (1) S ( ( 1 